DOI: $10.2478 / \mathrm{v} 10324-012-0002-7$<smiles></smiles>

VERSITA
Analele Universităţii de Vest,

Timişoara

Seria Matematică - Informatică

L, 1, (2012), 27- 37

\title{
Restricted Oriented Slack Convexity and Extremal Elements with Respect to a Set
}

Mihail Găianu and Gabriela Cristescu

\begin{abstract}
In a real linear space $V$, a set of directions $\mathcal{O} \subset V$ and a set of representative vectors $M \subset V$ generate a double restricted concept of convexity: with directional and with particular punctual restrictions. A Krein-Milman type property for slack $\mathcal{O}_{-}$ directionally convex sets with respect to $M$ is derived in terms of double restricted extremal elements of a set.
\end{abstract}

AMS Subject Classification (2000). 52A01, 52A05, 52C99.

Keywords. Convex hull, convex set, direction, extremal element, slack convexity.

\section{Introduction}

More domains of applied mathematics use procedures restrained to representative points of a set. For example, the integer programming is interested in points having integer coordinates, which means that $\mathbb{Z}^{n}$ represents $\mathbb{R}^{n}$ in this context [4], [12]. The rational programming restricts the procedures to $\mathbb{Q}^{n}$ instead $\mathbb{R}^{n}$ [12]. Image processing, image analysis and pattern recognition restricts the plane $\mathbb{R}^{2}$ to a set of grid points $\mathbb{Z}^{2}(h)=\{(i h, j h) \mid i \in \mathbb{Z}, j \in \mathbb{Z}\}$, with $h>0,[1],[4],[17]$. In a set of pixels isomorphic with $\mathbb{Z}^{2}(h)$, many tests are performed working on particular directions [17], [15], [14], [16]. The convexity property is a very useful one in all these domains of research and 
applications. Let us remind that, in a space $V$ in which a concept of straightline segment $\langle x, y\rangle$ joining any two elements $x, y \in V$ is defined, a subset $X \subseteq V$ is said to be convex if it contains the entire straight-line segment $\langle a, b\rangle$, whenever $a \in X$ and $b \in X$. There are many attempts to build models of convexity, either in digital framework [2], [7], [5], [9], or restricted to a set of representative points of a space [3], [11], [13], or restricting the orientation of straight-line segments [5], [15], [14], [16]. These directions of research, together with some practical applications [6] we faced with, are at the origin of our idea of double restricting the elements used in order to define the convexity: the elements of a set and the straight-line segments taken into account.

The framework of this paper is a real linear space $V$, in which two subsets are chosen: a subset of directions $\mathcal{O} \subseteq V$ and a subset of representative points $M \subseteq V$. The concept of slack $\mathcal{O}$-directionally convex set with respect to $M$, introduced in [5], is studied from the point of view of structural and algebraic properties. A representation theorem of Krein-Milman type [10], in a weaker form, is deduced in the framework of restricted directional convexity with respect to a given set. So, the facial structure [18] of sets with this kind of convexity is described in terms of $\mathcal{O}$-extremal points with respect to $M$.

\section{Slack $\mathcal{O}$-directional convexity with respect to a set}

Let $(V,+, \cdot)$ be a real linear space and $M \subseteq V$ a nonempty set of elements called representative points of $V$. Let $\mathcal{O} \subseteq V$ a set of vectors in $V$, called directions or orientations in $V$.

Remark 2.1. If the vectors of $\mathcal{O}$ are supposed to be linearly independent then the directions of the space are distinct. If its cardinality $|\mathcal{O}|=\operatorname{dim} V$, then $\mathcal{O}$ contains the directions of a basis in $V$.

Remark 2.2. If $\operatorname{dim} V=n$ and $\mathcal{O}$ is an orthogonal basis in $V$, then $\mathcal{O}$ contains a set of orthogonal directions in space. This is generally used in computational geometry, where the horizontal and vertical directions are preferred in various computations and testing procedures (see [17]).

Definition 2.1. A nonempty subset $X \subseteq V$ is said to be slackly $\mathcal{O}$-directionally convex with respect to $M$ if

$$
\{t x+(1-t) y \mid t \in[0,1]\} \cap M \subseteq X,
$$

whenever $x, y \in X \cap M$ such that there are $\alpha \in \mathbb{R}$ and $e \in \mathcal{O}$ having the property that $x-y=\alpha e$. 
The symbol $\mathcal{C}_{s}(\mathcal{O} ; M)$ is used, in the following, to denote the collection of all the subsets of $V$, which are slackly $\mathcal{O}$-directionally convex with respect to $M$. Let us remind that, according to [8], a pair $(S, \mathcal{K})$ is said to be a convexity space if $S$ is a nonempty set and $\mathcal{K}$ is a collection of subsets of $S$, which contains $\emptyset$ and $S$ and is closed for arbitrary intersection. The collection $\mathcal{K}$ is called a convexity in $S$.

Theorem 2.1. The pair $\left(V ; \mathcal{C}_{s}(\mathcal{O} ; M)\right)$ is a convexity space.

Proof. Obviously, $\varnothing \in \mathcal{C}_{s}(\mathcal{O} ; M)$ and $V \in \mathcal{C}_{s}(\mathcal{O} ; M)$. Let us suppose that $\mathcal{F} \subset \mathcal{C}_{s}(\mathcal{O} ; M)$. Denote by $F=\bigcap_{A \in \mathcal{F}} A$ and we shall prove that $F$ is slackly O-directionally convex with respect to $M$. Let $x \in F \cap M$ and $y \in F \cap M$ such that there are $k \in \mathbb{R}$ and $e \in \mathcal{O}$ such that $x-y=k e$. Then, $x \in A \cap M$ and $y \in A \cap M$, whenever $A \in \mathcal{F}$. Since $A$ is slackly $\mathcal{O}$-directionally convex with respect to $M$ it follows that

$$
\{t x+(1-t) y \mid t \in[0,1]\} \cap M \subseteq A,
$$

this inclusion being true for any $A \in \mathcal{F}$. As consequence,

$$
\{t x+(1-t) y \mid t \in[0,1]\} \cap M \subseteq \bigcap_{A \in \mathcal{F}} A=F,
$$

which means that $F$ is slackly $\mathcal{O}$-directionally convex with respect to $M$. So, $\mathrm{C}_{s}(\mathcal{O} ; M)$ is a convexity in $V$.

Q.E.D.

Example 2.1. Let $V=\mathbb{R}^{2}, M=\mathbb{Z}^{2}, \mathcal{O}=B_{2}=\left\{e_{1}, e_{2}\right\}$, with $e_{1}=(1,0)$ and $e_{2}=(0,1)$. The set $X=([1,3] \times\{1\}) \cup(\{1\} \times[1,2])$ is slackly $B_{2^{-}}$ directionally convex with respect to $\mathbb{Z}^{2}$. It is also integer convex according to the definition of Lupşa [12]. Also, it is ortho-convex in the sense defined by Rawlins and Wood [15], meaning that the straight-line segments with endpoints in $X$ lie in $X$ whenever they are either horizontal or vertical.

Example 2.2. Let $V=\mathbb{R}^{2}, M=\mathbb{Z}^{2}, \mathcal{O}=B_{2}=\left\{e_{1}, e_{2}\right\}$, with $e_{1}=(1,0)$ and $e_{2}=(0,1)$. The set $X=[1,3] \times[1,3] \backslash\{(2,2)\}$ is not slackly $B_{2}$-directionally convex with respect to $\mathbb{Z}^{2}$. Indeed, let $x=(1,2) \in X, y=(3,2) \in X$,

$$
\begin{gathered}
<x, y>=\{t x+(1-t) y \mid t \in[0,1]\}=[1,3] \times\{2\}, \\
<x, y>\cap M=\{(1,2),(2,2),(3,2)\} \nsubseteq X
\end{gathered}
$$

since $(2,2) \notin X$. 
Example 2.3. Let $V=\mathbb{R}^{2}, M=\mathbb{Z}^{2}, \mathcal{O}=B_{2}=\left\{e_{1}, e_{2}\right\}$, with $e_{1}=(1,0)$ and $e_{2}=(0,1)$. The set $X=\left\{(0,0),(0,1),(0,2),(1,0),(2,0),\left(\frac{3}{2}, 2\right)\right\}$ is slackly $B_{2}$-directionally convex with respect to $\mathbb{Z}^{2}$. Let us remark that the convexity requirement that the straight line segment having the endpoints in $X$ intersects $\mathbb{Z}^{2}$ in points belonging to $X$ is not valid. Indeed, choosing the points $x=(0,2)$ and $y=\left(\frac{3}{2}, 2\right)$ then $\langle x, y>\cap M=\{(0,2),(1,2)\} \nsubseteq X$ since $(1,2) \notin X$. As consequence, eliminating the condition $x, y \in X \cap M$ leads us to a different concept, stronger than that from definition 2.1.

Property 2.2. Let $\mathcal{O}_{1} \subseteq V, \mathcal{O}_{2} \subseteq V$ and $\mathcal{O}_{1} \subset \mathcal{O}_{2}$. Then

$$
\mathrm{e}_{s}\left(\mathcal{O}_{2} ; M\right) \subset \mathrm{e}_{s}\left(\mathcal{O}_{1} ; M\right) \text {. }
$$

Proof. If $X$ is slackly $\mathcal{O}_{2}$-directionally convex with respect to $M$ then for any $x, y \in X \cap M$, such that there are $e \in \mathcal{O}_{2}$ and $\alpha \in \mathbb{R}$ satisfying $x-y=\alpha e$, $<x, y>\cap M \subset X$. Let $x, y \in X \cap M$ and let us suppose that there is $f \in \mathcal{O}_{1}$ such that $x-y=\beta f$, for a number $\beta \in \mathbb{R}$. Since $\mathcal{O}_{1} \subset \mathcal{O}_{2}$ it follows that $f \in \mathcal{O}_{2}$. So, due to the slack $\mathcal{O}_{2}$-directional convexity of $X$ with respect to $M$ it follows that $\langle x, y\rangle \cap M \subset X$, which proves the slack $\mathcal{O}_{1}$-directional convexity of $X$ with respect to $M$.

Q.E.D.

Property 2.3. Let $\mathcal{O} \subseteq V, M_{1} \subseteq V, M_{2} \subseteq V$ and $M_{1} \subset M_{2}$. Then

$$
\mathcal{C}_{s}\left(\mathcal{O} ; M_{2}\right) \subset \mathcal{C}_{s}\left(\mathcal{O} ; M_{1}\right)
$$

Proof. If $X$ is slackly $\mathcal{O}$-directionally convex with respect to $M_{2}$ then for any $x, y \in X \cap M_{2}$, such that there are $e \in \mathcal{O}$ and $\alpha \in \mathbb{R}$ satisfying $x-y=\alpha e$, $<x, y>\cap M_{2} \subset X$. Let $x, y \in X \cap M_{2}$ and let us suppose that there is $f \in \mathcal{O}$ such that $x-y=\beta f$, for a number $\beta \in \mathbb{R}$. Since $M_{1} \subset M_{2}$ and due to the slack $\mathcal{O}$-directional convexity of $X$ with respect to $M_{2}$ it follows that

$$
<x, y>\cap M_{1} \subset<x, y>\cap M_{2} \subset X,
$$

which proves the slack $\mathcal{O}$-directional convexity of $X$ with respect to $M_{1}$. Q.E.D.

Let $\alpha \in \mathbb{R}^{*}$. Like in [4] we say that a set $M \subseteq V$ is an $\alpha$-set if $\frac{1}{\alpha} x \in M$ for any $x \in M$.

Example 2.4. For example, every cone in a vectorial space is an $\alpha$-set, whenever $\alpha \in \mathbb{R}_{+}^{*}$.

Example 2.5. The set $\mathbb{Z}$ of all integer numbers is a (-1)-set. 
Example 2.6. The set $\mathbb{Q}$ of all rational numbers is an $\alpha$-set, whenever $\alpha \in \mathbb{Q}^{*}$.

Property 2.4. If $M \subseteq V$ is a nonempty $\alpha$-set and $X \subseteq V$ is slackly $\mathcal{O}$ directionally convex with respect to $M$ then $\alpha X$ is slackly $\mathcal{O}$-directionally convex with respect to $M$.

Proof. Let us suppose that $X$ is slackly $\mathcal{O}$-directionally convex with respect to $M$. Let $x \in \alpha X \cap M$ and $y \in \alpha X \cap M$ such that $x-y=k e$ for a number $k \in \mathbb{R}$ and a vector $e \in \mathcal{O}$. Then there are $u \in X$ and $v \in X$ such that $x=\alpha u$ and $y=\alpha v$. Then one has

$$
u-v=\frac{1}{\alpha}(x-y)=\frac{k}{\alpha} e
$$

and, from the slack $\mathcal{O}$-directional convexity of $X$ with respect to $M$ it follows that

$$
\{t u+(1-t) v \mid t \in[0,1]\} \cap M \subseteq X .
$$

Let us suppose that for a number $t_{1} \in[0,1]$, one has $t_{1} u+\left(1-t_{1}\right) v \in M$. Since $M$ is an $\alpha$-set, it follows that $\alpha\left[t_{1} u+\left(1-t_{1}\right) v\right] \in M$. But

$$
\alpha\left[t_{1} u+\left(1-t_{1}\right) v\right]=t_{1}(\alpha u)+\left(1-t_{1}\right) \alpha v=t_{1} x+\left(1-t_{1}\right) y \in M .
$$

Since $X$ is slackly $\mathcal{O}$-directionally convex with respect to $M$ one gets $t_{1} u+$ $\left(1-t_{1}\right) v \in X$. Then

$$
\alpha\left[t_{1} u+\left(1-t_{1}\right) v\right]=t_{1} x+\left(1-t_{1}\right) y \in \alpha X
$$

Therefore, $\alpha X$ is slackly $\mathcal{O}$-directionally convex with respect to $M$. Q.E.D.

\section{Slack O-directional convex hull with respect to $M$}

As it is well known from the classical theory of convexity ([7] and [18]) the convex hull of a set $S \subset V$ is given, using the outer characterization, by the intersection of all the convex sets including $S$. The inner geometric approach describes the convex hull of $S$ as the union of all the straight-line segments joining points of $S$. Similarly one may introduce the slack $\mathcal{O}$-directionally convex hull with respect to $M$ of a set $S \subset V$. 
Definition 3.1. If $S \subset V$ then the set

$$
\mathcal{O}_{M}^{(s)} \operatorname{conv}(S)=\bigcap\left\{P \subset V \mid S \subseteq P \text { and } P \in \mathcal{C}_{s}(\mathcal{O} ; M)\right\}
$$

is called the slack $\mathcal{O}$-directionally convex hull with respect to $M$ of $S$.

If $A \subset V$, let us denote by $\left.A\right|_{\mathcal{O}}$ the set of all those points $x \in A$ that have the property that there is at least one point $y \in A$ such that $y-x=k e$ for some $k \in \mathbb{R}$ and $e \in \mathcal{O}$. It means that each point of $\left.A\right|_{\mathcal{O}}$ is an extremity of a straight-line segment lying in $A$ and having a direction from $\mathcal{O}$.

Theorem 3.1. A set $X \subseteq V$ is slackly $\mathcal{O}$-directionally convex with respect to $M$ if and only if

$$
M \cap \mathcal{O}_{M}^{(s)} \operatorname{conv}(X)=M \cap X .
$$

Proof . Necessity. Let us suppose that $X$ is slackly $\mathcal{O}$-directionally convex with respect to $M$. From definition 2.1 one gets that

$$
M \cap \mathcal{O}_{M}^{(s)} \operatorname{conv}(X) \subseteq X
$$

But $M \cap \mathcal{O}_{M}^{(s)} \operatorname{conv}(X) \subseteq M$, so it follows that

$$
M \cap \mathcal{O}_{M}^{(s)} \operatorname{conv}(X) \subseteq M \cap X .
$$

Since $X \cap M \subseteq \mathcal{O}_{M}^{(s)} \operatorname{conv}(X)$, one has

$$
M \cap X \subseteq M \cap \mathcal{O}_{M}^{(s)} \operatorname{conv}(X) .
$$

From (3.3) and (3.4) one gets

$$
M \cap X=M \cap \mathcal{O}_{M}^{(s)} \operatorname{conv}(X) .
$$

Sufficiency Let $X \cap M \subseteq \mathcal{O}_{M}^{(s)} \operatorname{conv}(X)$. It follows that $X \cap M$ is slackly O-directionally convex with respect to $M$. Then, from definition 2.1 , one gets that

$$
\left.M \cap[\operatorname{conv}(M \cap X)]\right|_{\mathcal{O}} \subseteq X \cap M .
$$

This means that

$$
\left.M \cap[\operatorname{conv}(M \cap X)]\right|_{\mathcal{O}} \subseteq X \cap M \subseteq X,
$$

therefore $X$ is slackly $\mathcal{O}$-directionally convex with respect to $M$. 


\section{Extremal elements of slack O-directionally convex sets with respect to $M$}

Let $V$ be a vectorial space, $\mathcal{O} \subseteq V$ a set of directions in $V$ and a set $M \subseteq V$, $\mathcal{O} \neq \emptyset, M \neq \emptyset$.

Definition 4.1. An element $x \in X \cap M$ is said to be an $\mathcal{O}$-extremal point of $X$ with respect to $M$ if there are no two distinct points $y_{1}, y_{2} \in(X \backslash\{x\}) \cap M$, with $y_{1}-y_{2}=\lambda e$ for a number $\lambda \in \mathbb{R}$ and a direction $e \in \mathcal{O}$, and there is no number $t \in[0,1]$ such that the following conditions simultaneously hold:

$$
\begin{gathered}
t y_{1}+(1-t) y_{2} \in M \\
t y_{1}+(1-t) y_{2}=x
\end{gathered}
$$

Corollary 4.1. An element $x \in X \cap M$ is an $\mathcal{O}$-extremal point of $X$ with respect to $M$ if

$$
\left.x \notin M \cap[\operatorname{conv}((X \backslash\{x\}) \cap M)]\right|_{\mathcal{O}} .
$$

Let us denote by $\mathcal{O}_{M} \operatorname{Exr}(X)$ the set of all $\mathcal{O}$-extremal points of the set $X$ with respect to $M$.

Example 4.1. If $V=\mathbb{R}, M=Q, \mathcal{O}=\{1\}$ and $X=\{x \in Q \mid-\sqrt{2} \leq x \leq$ $\sqrt{2}\}$ then it is obvious that $\mathcal{O}_{M} \operatorname{Exr}(X)=\emptyset$.

Example 4.2. If $V=\mathbb{R}, M=Q, \mathcal{O}=\{1\}$ and $X=\{0,1,3\}$ then $\mathcal{O}_{M} \operatorname{Exr}(X)=\{0,3\}$.

Example 4.3. If $V=\mathbb{R}^{2}, M=\mathbb{Z}^{2}, \mathcal{O}=\{(0,1),(1,0),(1,1),(-1,1)\}$ and $X=\{(0,2),(-1,1),(1,0),(-0,1)\}$ then $\mathcal{O}_{M} \operatorname{Exr}(X)=X$.

Theorem 4.2. If $X \subseteq V, \mathcal{O} \subseteq V, M \subseteq V, M \neq \emptyset$, and the set $\mathcal{O} \neq \emptyset, X$ is slackly $\mathcal{O}$-directionally convex with respect to $M$ then the following sentences are equivalent:

1. $x \in \mathcal{O}_{M} \operatorname{Exr}(X)$;

2. if there are two elements $y_{1}, y_{2} \in X \cap M$, with $y_{1}-y_{2}=\lambda e$, for $a \lambda \in \mathbb{R}$ and $e \in \mathcal{O}$, such that

$$
x \in M \cap \mathcal{O}_{M}^{s} \operatorname{conv}\left\{y_{1}, y_{2}\right\},
$$

then it follows that $y_{1}=y_{2}=x$; 
3. the set $X \backslash\{x\}$ is slackly $\mathcal{O}$-directionally convex with respect to $M$.

Proof . 1) $\Rightarrow 2$ ) Let us suppose that sentence 1) is true and sentence 2) is false. Then there are two distinct elements $y_{1}, y_{2} \in X \cap M$, situated on one of the directions from $\mathcal{O}$ and there is a number $t \in[0,1]$ such that

$$
x=t y_{1}+(1-t) y_{2} .
$$

- If $y_{1}=y_{2}=x$ then 2) is true.

- Otherwise, it is possible that one of the points $y_{1}$ or $y_{2}$ coincides with $x$. Let us suppose that $y_{1}=x$ and $y_{2} \neq x$. Then

$$
(1-t) x=(1-t) y_{2}
$$

and dividing by $(1-t)$ one gets $y_{2}=x$, which is a contradiction. So, sentence 2) is true.

- It is possible that both $y_{1} \neq x$ and $y_{2} \neq x$. In this case, let us chose $z=x$. One gets the elements $y_{1}$ and $z$, with $y_{1} \neq x, z=x$ and there is $t \in[0,1]$ such that $t z+(1-t) y_{1}=x$, which reduces to the previous one. Analogously, if one chooses $z=x$ and $y_{2} \neq x$.

As consequence, proposition 2) is true.

$2) \Rightarrow 3$ ) Let us suppose that proposition 2) is true and 3) is false. Then there are $y_{1}, y_{2} \in(X \backslash\{x\}) \cap M$, situated on one of the directions from $\mathcal{O}$ and there is a number $t \in[0,1]$ such that

$$
u=t y_{1}+(1-t) y_{2} \in M
$$

and $u \notin X \backslash\{x\}$. From the property of slackly $\mathcal{O}$-directional convexity with respect to $M$ of set $X$ it follows that $u \in X$. As consequence, from 2) one deduces that $u=x=y_{1}=y_{2}$, which represents a contradiction with the condition $y_{1}, y_{2} \in X \backslash\{x\}$. Therefore, 3$)$ is true.

$3) \Rightarrow 1$ ) If the set $X \backslash\{x\}$ is slackly $\mathcal{O}$-directionally convex with respect to $M$ then the element $x$ satisfies the conditions of an $\mathcal{O}$-extremal point of $X$ with respect to $M$, in an obvious manner.

Q.E.D

Let us consider a subset $S \subset V$. The set $(\mathcal{O}, M)-\operatorname{Core}(S)=\{\bigcup\{<x, y\rangle$ $\mid x \in S \bigcap M, y \in S \bigcap M, \exists k \in \mathbb{R}, \exists e \in \mathcal{O}, y-x=k e\}\} \bigcap S$ is called the slack O-directional core of $S$ with respect to $M$.

Lemma 4.3. If $X \subseteq V, \mathcal{O} \subseteq V, M \subseteq V$, and $\mathcal{O} \neq \emptyset$ and $M \neq \emptyset$, than

$$
\mathcal{O}_{M} \operatorname{Exr}(X) \supset \mathcal{O}_{M} \operatorname{Exr}((\mathcal{O}, M)-\operatorname{core}(X)) .
$$


Proof . Let $x \in \mathcal{O}_{M} \operatorname{Exr}((\mathcal{O}, M)-\operatorname{core}(X))$. It means that there are not two points $y_{1}, y_{2} \in(X \backslash\{x\}) \cap M$ such as there are $\lambda \in \mathbb{R}$ and $e \in \mathcal{O}$ with $y_{1}-y_{2}=\lambda e$ and there is not $t \in[0,1]$ such as simultaneously $t y_{1}+(1-t) y_{2} \in$ $M$ and $t y_{1}+(1-t) y_{2}=x$. It means that $x \in \mathcal{O}_{M} \operatorname{Exr}(X)$.

Q.E.D

Remark 4.1. The converse inclusion does not hold, since every element of $X \cap M$ that does not belong to $(\mathcal{O}, M)-\operatorname{core}(X)$ is an $\mathcal{O}$-extremal point with respect to $M$ of $X$.

Theorem 4.4. If $X \subset V$ is slackly $\mathcal{O}$-directionally convex with respect to $M$ then

$$
X \cap M=\left\{\mathcal{O}_{M}^{s} \operatorname{conv}\left(\mathcal{O}_{M} \operatorname{Exr}(X)\right)\right\} \cap M
$$

Proof . Let us suppose that $x \in X \cap M$. Then two cases are possible:

- $x \in(\mathcal{O}, M)-\operatorname{core}(X)$, which means that $x \in\left\{\mathcal{O}_{M}^{s} \operatorname{conv}\left(\mathcal{O}_{M} \operatorname{Exr}((\mathcal{O}, M)-\right.\right.$ core $(X)))\} \cap M \subset\left\{\mathcal{O}_{M}^{s} \operatorname{conv}\left(\mathcal{O}_{M} \operatorname{Exr}(X)\right)\right\} \cap M$, according to lemma 4.3 .

- $x \notin(\mathcal{O}, M)-\operatorname{core}(X)$, which means that $x$ is an $\mathcal{O}$-extremal point of $X$ with respect to $M$ and then $x \in\left\{\mathcal{O}_{M}^{s} \operatorname{conv}\left(\mathcal{O}_{M} \operatorname{Exr}(X)\right)\right\} \cap M$. It follows that $x \cap M \subseteq\left\{\mathcal{O}_{M}^{s} \operatorname{conv}\left(\mathcal{O}_{M} \operatorname{Exr}(X)\right)\right\} \cap M$.

Conversely, let us suppose that $x \in\left\{\mathcal{O}_{M}^{s} \operatorname{conv}\left(\mathcal{O}_{M} \operatorname{Exr}(X)\right)\right\} \cap M$. It follows that there are two elements $y_{1}, y_{2} \in \mathcal{O}_{M} \operatorname{Exr}(X)$ and $z_{1}, z_{2} \in \mathcal{O}_{M} \operatorname{Exr}(X)$ and two elements $a_{1} \in<z_{1}, z_{2}>\cap M$ and $a_{2} \in<z_{1}, z_{2}>\cap M$ such as $x \in<a_{1}, a_{2}>\cap M$ (more similar steps may be necessary). But $X$ is slackly $\mathcal{O}_{-}$ directionally convex with respect to $M$, implying that $a_{1} \in X \cap M, a_{2} \in X \cap$ $M$ and, as consequence, $x \in X \cap M$. It implies that $\left\{\mathcal{O}_{M}^{s} \operatorname{conv}\left(\mathcal{O}_{M} \operatorname{Exr}(X)\right)\right\} \cap$ $M \subseteq X \cap M$. The theorem is completely proved.

Q.E.D

Remark 4.2. Theorem 4.4 is a weaker result than the classical Krein-Milman theorem [10]. It is the best possible result of Krein-Milman type in the framework of the slack $\mathcal{O}$-directional convexity with respect to a given set $M$.

\section{References}

[1] J. M. Chassery, Représentation discrète, interprétation numérique et description des images: des concepts à l'applications, Thèse, Université Scientifique et Médicale de Grenoble, 1984. 
[2] G. Cristescu, Convexity related to the coarseness of the concavity, Révue d'Analyse Num. Th. Approx., 26, 1-2, (1997), 29-37.

[3] G. Cristescu, Extinderea noţiunilor de comportare şi aproximare la unele domenii particulare ale cercetării, Thesis, Universitatea "Babeş - Bolyai“ din Cluj-Napoca, 1997.

[4] G. Cristescu and L. Lupşa, Non-Connected Convexities and Applications, Kluwer Academic Publishers, Dordrecht/ Boston/ London, 2002.

[5] M. Găianu, Contribuţii la studiul unor probleme de recunoaştere şi regăsire de imagini, Thesis, Universitatea de Vest din Timişoara, 2011.

[6] M. Găianu, Detecting and tracking markers on a human face, Annals of the Oradea University, Fascicle of Management and Technological Engineering, X(X), 1(2011), 3.49-3.54.

[7] P. M. Gruber and J. M. Willis, (eds.), Handbook of Convex Geometry, A,B, North-Holland, 1993.

[8] D. C. Kay and E. W. Womble, Axiomatic convexity theory and relationships between the Caratheodory, Helly and Radon numbers, Pacific J. Math., 38, (1971), 471-485.

[9] C. E. Kim and A. Rosenfeld, On the convexity of digital regions, Proc. 5th JCPR, (1980), 1010-1015.

[10] M. Krein and D. Milman, On the extreme points of regularly convex sets, Studia Mathematica, 9, (1940), 133-138.

[11] L. Lupşa, Asupra unei noţiuni de convexitate, Sem. Itin. Ec. Funcţ. Aprox. Conv., 7-8 noiembrie 1980, (1980), 127-135.

[12] L. Lupşa, Tipuri particulare de probleme de programare liniară şi neliniară, Teză de doctorat, Universitatea "Babeş-Bolyai" Cluj-Napoca, 1981.

[13] L. Lupşa, Some types of convexity with respect to a given set, (1986), 89-114.

[14] G. J. E. Rawlins and D. Wood, Optical Computation of Finitely-Oriented Convex Hulls, Information and Computation, 72, (1987), 150-166.

[15] G. J. E. Rawlins and D. Wood, Ortho-convexity and its generalizations, Computational Morphology, G.T. Toussaint ed., Elsevier, (1988), 137-152.

[16] G. J. E. Rawlins and D. Wood, Restricted-Oriented Convex Sets, Technical Report, 272, 1989.

[17] A. Rosenfeld, Picture Processing by Computer, Academic Press, New York, London, 1969.

[18] R. Webster, Convexity, Oxford University Press, 1994.

Mihail Găianu

West University of Timişoara

Timişoara

Romania 
Vol. L (2012) Restricted Oriented Slack Convexity and ...

E-mail: mgaianu@info.uvt.ro

Gabriela Cristescu

University "Aurel Vlaicu" of Arad

Arad

Romania

E-mail: gcristescu@inext.ro

Received: 05.01.2012

Accepted: 11.06.2012 